\title{
Propylthiouracil-coated biodegradable polymer inhibited neointimal formation and enhanced re-endothelialization after vascular injury
}

This article was published in the following Dove Press journal:

International Journal of Nanomedicine

\author{
Shang-Hung Chang' \\ Cheng-Hung Lee' \\ Yung-Hsin Yeh' \\ Shih-Jung Liu ${ }^{2}$ \\ Chao-Jan Wang ${ }^{3}$ \\ Ming-Yi $\mathrm{Hsu}^{3}$ \\ Wei-Jan Chen'
}

'Cardiovascular Department, Chang Gung Memorial Hospital, Chang-

Gung University College of Medicine,

Tao-Yuan, Taiwan; ${ }^{2}$ Department

of Mechanical Engineering, Chang

Gung University, Tao-Yuan, Taiwan;

${ }^{3}$ Department of Medical Imaging and Intervention, Chang Gung Memorial

Hospital, Tao-Yuan, Taiwan
Correspondence: Wei-Jan Chen

Cardiovascular Department, Chang

Gung Memorial Hospital, Chang-Gung

University College of Medicine,

Chang-Gung University, Fu-Shin

Road Number 5, Kwei-Shan,

Tao-Yuan 333, Taiwan

Tel +886 $3328 \quad 1200$ ext 8162

Fax +8863327 I I 92

Email wjchen@adm.cgmh.org.tw
Background: The drug-eluting stent is a standard approach for the treatment of coronary artery disease. Propylthiouracil (PTU), an antithyroid drug, has been proven to suppress neointimal formation after balloon injury.

Materials and methods: This study used a biodegradable polymer coating with PTU to test its effects on platelet function, re-endothelialization, and neointimal formation after vascular injury. Electrospinning was used to fabricate hybrid stents and generate PTU-loaded nanofibers. Results: PTU-eluting stents maintained a stable release of PTU for 3 weeks. The PTU-coated stent markedly decreased the neointimal formation induced by vascular injury in the descending aorta of rabbits. Moreover, the PTU coating reduced platelet adhesion on the surface of the biodegradable membrane, which was reflected by the decreased expression of adhesion molecule in PTU-treated endothelial cells. The PTU coating enhanced re-endothelialization in injured aortas. In vitro, PTU exerted less suppressive effect on the proliferation and migration of endothelial cells than on those of vascular smooth muscle cells. Furthermore, treatment of endothelial cells with PTU induced phosphorylation (Ser1177) of endothelial nitric oxide synthase as well as its association with heat shock protein 90, supporting the protective role of PTU in endothelial function. The level of thyroid-stimulating hormone remained unchanged during the experimental period.

Conclusion: This study indicates that PTU can be released locally and steadily in injured aortas, with some local effects but without systemic effects. Furthermore, PTU-coated stents may have beneficial effects on neointimal formation, endothelial cell, and platelet functions.

Keywords: drug-eluting stent, propylthiouracil, neointimal formation, endothelial cell, platelet

\section{Introduction}

Percutaneous coronary intervention with drug-eluting stents is a well-established method for the treatment of coronary artery disease. ${ }^{1}$ Stents coated with sirolimus have been proven to prevent neointimal formation/restenosis after stent deployment, which is mainly via its inhibitory effect on the proliferation of vascular smooth muscle cell (VSMC). ${ }^{1,2}$ However, because sirolimus could obstruct the mobilization and differentiation of endothelial cells, sirolimus-coated stents might impair re-endothelialization and increase the incidence of late stent thrombosis. ${ }^{3,4}$ Clinically, the main strategy in preventing late stent thrombosis is to prolong dual antiplatelet therapy, which might impart the risk of lethal bleeding. ${ }^{5-7}$ Furthermore, long-term antiplatelet usage is still problematic in certain clinical conditions, such as patients who have recently undergone surgery or have coagulopathy or recent stroke, wherein they suffer from acute coronary syndromes when preparing for coronary interventions. ${ }^{5-7}$ Conceivably, 
there is a need to develop potential drugs that could overcome the abovementioned hurdles.

Propylthiouracil (PTU), independent of its antithyroid effect, has also been found to possess anti-atherosclerotic properties. $^{8}$ Our previous studies demonstrated that PTU could inhibit VSMC proliferation, modulate the VSMC phenotype, and suppress neointimal formation in balloon-injured rat carotid arteries. ${ }^{8-10}$ Accordingly, PTU may be applicable in the clinic for the prevention of restenosis after balloon injury. However, systemic administration of PTU is impractical because it would result in hypothyroidism. To prohibit this systemic effect, this study used a biodegradable polymer poly(D,L)-lactide-co-glycolide (PLGA) - for coating a commercially available stent together with PTU to test its potential effects on platelet adhesion, re-endothelialization, and neointimal formation after vascular injury. PLGA is a synthesized, biodegradable, noncytotoxic copolymer, and it can constitute a stable and locally released system without accumulating in vital organs. ${ }^{11}$ Moreover, the in vivo observation was verified in cultured VSMCs and endothelial cells.

\section{Materials and methods Production of PTU-coated stent}

The commercially available PLGA (Resomer RG 503, Boehringer, Mainz, Germany) in this study contained a lactide:glycolide ratio of 50:50 with a molecular weight of $\sim 33,000 \mathrm{Da}$, as measured by a gel permeation chromatograph equipped with a Waters 2414 Refractive Index Detector. PTU and 1,1,1,3,3,3-hexafluoro-2-propanol (HFIP) were purchased from Sigma-Aldrich (St Louis, MO, USA). The electrospinning setup of this study consisted of a syringe and needle (internal diameter $0.42 \mathrm{~mm}$ ), ${ }^{11}$ a ground electrode, a metallic pin (diameter $0.95 \mathrm{~mm}$ ) mounted on a motor, and a high-voltage power source. The needle and metallic pin were connected to the high-voltage power source, which generated positive direct current voltages and currents of up to $35 \mathrm{kV}$ and $4.16 \mathrm{~mA} / 125 \mathrm{~W}$, respectively. The motor was set to rotate at $10 \times g$. To electrospin the nanofibers, a predetermined weight ratio of PLGA and PTU (240 mg/40 mg, w/w) was first dissolved in $1 \mathrm{~mL}$ HFIP. The solution was then delivered and electrospun by a syringe pump with a volumetric flow rate of $3.6 \mathrm{~mL} /$ hour to obtain nanofibrous tubes on a metallic pin. The distance between the needle tip and the ground electrode was $10 \mathrm{~cm}$, and the positive voltage applied to the polymer solutions was $17 \mathrm{kV}$. All electrospinning experiments were conducted at room temperature. After electrospinning, we crimped the electrospun nanofibrous tube on the outside of commercially available Gazella bare-metal stents (each measuring $2.75 \times 11 \mathrm{~mm}^{2}$, Biosensors International, Morges, Switzerland) by hand to produce hybrid PTU nanofiber-mounted stents. All of these stents were placed in a vacuum oven at $40^{\circ} \mathrm{C}$ for 72 hours to evaporate the solvents before use.

\section{Platelet adhesion at nanofibers}

Blood from a healthy rabbit was mixed with $3.2 \%$ sodium citrate at a ratio of $9: 1 \mathrm{blood} / \mathrm{sodium}$ citrate at $22^{\circ} \mathrm{C}$. Plateletrich plasma (PRP) was obtained from the blood mixture by centrifugation at $150 \mathrm{~g}$ for 10 minutes. The platelet concentration in the PRP was $2 \times 10^{5}$ cells $/ \mu \mathrm{L}$ as measured by a semi-automated hematology analyzer (SYSMEX F820). The platelet suspension $\left(50 \mu \mathrm{L} ; \sim 1 \times 10^{7}\right.$ platelets $)$ was incubated on the surfaces of nanofibers that were either plain or coated with PTU at $37^{\circ} \mathrm{C}$ for 3 hours. The fibers were then washed three times with phosphate-buffered saline (PBS), fixed with $1 \%$ glutaraldehyde, immersed in PBS, and placed to stand in a fixature for 60 minutes at $4^{\circ} \mathrm{C}$.

\section{In vitro release of PTU}

An elution method was used to detect the in vitro release of PTU from the nanofiber. The nanofiber coated with PTU $\left(25 \mu \mathrm{g} / \mathrm{mm}^{2}\right)$ was placed in a glass test tube that contained $1 \mathrm{~mL}$ PBS $(0.15 \mathrm{~mol} / \mathrm{L}, \mathrm{pH} 7.4$; one sample per test tube) at $37^{\circ} \mathrm{C}$ for 24 hours. The eluent was then collected and analyzed daily for 28 days.

The PTU concentration in the eluent was determined using a high-performance liquid chromatography (HPLC) assay as described. ${ }^{8}$ The HPLC analysis was conducted on a Hitachi L-2200 Multisolvent Delivery System. A SYMMETRY $\mathrm{C}_{8}$ (3.9 $\mathrm{cm} \times 150 \mathrm{~mm}$ HPLC column; Waters) was used for the separation of PTU. The mobile phase contained $0.01 \mathrm{~mol} / \mathrm{L}$ heptanesulfonic acid (Fisher Scientific U.K. Ltd.) and acetonitrile (85/15, v/v; Mallinckrodt, St. Louis, MO, USA). Absorbency was monitored at a wavelength of $254 \mathrm{~nm}$ with a flow rate of $1.4 \mathrm{~mL} / \mathrm{min}$. All experiments were conducted in triplicate, and the sample dilutions brought the unknown concentrations into the range of a standard curve. A calibration curve was made for each set of measurements (correlation coefficient $>0.99$ ). The elution product was identified and quantified with a high degree of sensitivity using the HPLC system.

\section{Surgical procedure and animal care}

Adult, male, New Zealand white rabbits $(n=24$; mean weight of $3.5 \pm 0.3 \mathrm{~kg}$ ) were housed in individual cages in a temperature- and light-controlled room and given standard rabbit chow ad libitum with free access to sterilized 
drinking water. All animal procedures had been institutionally approved, and all of the studied animals were cared under the supervision of a licensed veterinarian according to the regulations of the National Institute of Health of Taiwan. All animal experiments were reviewed and approved by the Chang Gung Memorial Hospital (permit no 2015050601).

Rabbits were sedated and anesthetized by a muscular injection of xylazine $(9.3 \mathrm{mg} / \mathrm{kg})$, and by administering Zoletil 50 ${ }^{\mathrm{TM}}$ (tiletamine-zolazepam $10 \mathrm{mg} / \mathrm{kg}$ ) and oxygen ( $2 \mathrm{~L} / \mathrm{min}$ ) through a face mask. A $5 \mathrm{~F}$ sheath was inserted into the right femoral artery using the puncture technique. Hybrid stents on which nanofibers were loaded with PTU $\left(25 \mu \mathrm{g} / \mathrm{mm}^{2}\right.$ with total doses of $1,250 \mu \mathrm{g}$ ) were mounted.

Rabbits were separated into two groups: Group A ( $n=12)$ received hybrid stents loaded with PTU and Group B $(n=12)$ received hybrid stents without drug loading. Rabbits firstly underwent endothelial denudation using a $3.5 \times 20 \mathrm{~mm}$ Maverick $^{\mathrm{TM}}$ balloon (Boston Scientific, Maple Grove, MN, USA) in the descending abdominal aorta to cause a balloon injury. The balloon was passed over a 0.014 -inch guide wire, moved into the thoracic aorta, inflated to nominal pressure ( 8 bars with $50 \%[\mathrm{v} / \mathrm{v}]$ contrast/saline), and withdrawn in a retrograde manner to the low descending abdominal aorta. The same procedure was repeated three times. Hybrid stents were then deployed in the descending abdominal aorta. Postprocedural angiography was undertaken to document the vessel patency. To comply with institutional regulations, all rabbits were administered aspirin ( $40 \mathrm{mg} /$ day) orally 24 hours before the catheterization and this was continued throughout the in-life phase. Furthermore, a single dose of intra-arterial heparin $(150 \mathrm{IU} / \mathrm{kg})$ was administered upon catheterization.

The stented vessels were collected at $3(n=6)$ and 8 weeks $(n=6)$ after deployment and sent for microscopic and histological examinations. Each anesthetized animal was exsanguinated by left ventricular puncture, perfused with lactated Ringer's solution, and given a lethal dose of lidocaine $(100 \mathrm{mg} / \mathrm{kg})$. Explanted vessels were flushed with lactated Ringer's solution, fixed, transversely sectioned, and stained with hematoxylin and eosin. The intimal thickness was measured for each cross section at the middle of the stenting area and recorded from 24 stented arteries in 12 animals at Week 8.

\section{Microscopic observation}

The stented vessels were longitudinally bisected to expose the luminal surface and photographed. Vessels were rinsed with $0.1 \mathrm{mmol} / \mathrm{L}$ sodium phosphate buffer $(\mathrm{pH} 7.2 \pm 0.1)$ and subsequently fixed in 1\% osmium tetroxide for 30 minutes. Vessels were then dehydrated in a graded series $(50 \%$,
$60 \%, 70 \%, 80 \%, 90 \%$, and $100 \%$ ) of ethanol. After drying, vessels were mounted, sputter-coated with gold, and observed under a scanning electron microscope (SEM; Hitachi S-3000N, Tokyo, Japan).

Low-power photographs of the luminal surface, magnified $35 \times$, were taken to estimate the degree of endothelialization at the implant. Serial en face SEM images were obtained at low power $(35 \times)$ and digitally assembled to generate a complete view of the entire stent surface. The images were further enlarged $(1,000 \times)$ for direct visualization of the endothelial cells. The extent of the endothelial surface coverage above the stent struts was traced and measured using ImageJ software (National Institutes of Health, Bethesda, MD, USA). ${ }^{12}$ At least five random areas were chosen to calculate the degrees of cell coverage and expressed as endothelial coverage percentages.

\section{Histological examination}

A semi-quantitative scoring approach was used to evaluate the degree of inflammation and vascular injury ${ }^{13}$ - briefly, inflammation score $(0=$ none; $1=$ mild, including minimal infiltrated inflammatory cells; $2=$ moderate; and $3=$ severe with large clusters of inflammatory cells with granulomatous morphology); vessel injury score $(0=$ strut not in contact with internal elastic lamina [IEL]; $1=$ strut in contact with IEL and profile in the neointima; $2=$ strut penetrates IEL with profile in media; $3=$ strut penetrates media and is in contact with external elastic lamina; and $4=$ strut is in the adventitia). The score for each strut was averaged to obtain the mean score for each of the 72 histological sections from 24 stented arteries in 12 animals at Week 8.

\section{Western blotting}

Western blotting was undertaken as described previously. ${ }^{8-10}$ Equal amounts of protein in the sodium dodecyl sulfatepolyacrylamide gel electrophoresis (SDS-PAGE) sample buffer was sonicated and subjected to electrophoresis on $8 \%$ SDS-polyacrylamide gels. After being transferred to polyvinylidene difluoride membranes (Stratagene, Amsterdam, the Netherlands), proteins were incubated with primary antibodies against the platelet and endothelial cell adhesion molecule-1 (PECAM-1, Abcam \#124432, Cambridge, UK), phospho-endothelial nitric oxide synthase (p-eNOS; Ser1177, Abcam \#184154), endothelial nitric oxide synthase (eNOS; BD \#610299, Franklin, NJ, USA), and tubulin (Santa Cruz Biotechnology, Dallas, TX, USA). Signals were detected by ECL-detection (Amersham, the Netherlands) and quantified by densitometry. 


\section{Thyroid function measurement}

The thyroid function of the rabbits was measured with a Rabbit Thyroid Stimulating Hormone (TSH) ELISA Kit (Cubasio) according to the manufacturer's instructions. Sera of rabbits were collected both before and 8 weeks after stenting.

\section{Cell culture}

Human VSMCs and umbilical vein endothelial cells (HUVECs) were purchased from the American Type Culture Collection (PCS-100-101 ${ }^{\mathrm{TM}}$ ). In all experiments, cells between the fourth and seventh passage were used and cultured on conventional uncoated dishes. PTU was dissolved in $100 \%$ dimethysulfoxide (DMSO) at final concentrations of $0.05-5 \mathrm{mmol} / \mathrm{L}$. Cells were treated with $1 \%$ DMSO as a vehicle.

\section{Cell proliferation assay}

The proliferative activities of VSMCs and endothelial cells were determined by a BrdU incorporation assay. ${ }^{14,15}$ After 24 hours of serum deprivation, cells were incubated with BrdU for 16 hours. Incorporation of BrdU into cells was detected by an ELISA detecting kit (Roche, Mannheim, Germany) according to the manufacturer's instructions.

\section{Cell migration assay}

A Transwell filter chamber (Corning Costar) with 8.0- $\mu \mathrm{m}$ pore size was used for migration assay. Cells were seeded at a density of $5 \times 10^{5}$ cells per filter. To initiate the chemotaxis assay, cells in $200 \mu \mathrm{L}$ Dulbecco's Modified Eagle's Medium (DMEM) without FBS were added to the upper chamber, and the bottom chamber was filled with $600 \mu \mathrm{L}$ DMEM plus $10 \% \mathrm{FBS}$ as chemotaxis factor for cell movement. Cells were allowed to migrate at $37^{\circ} \mathrm{C}$ for 6 hours. Cells on the lower aspect of the filter membrane were stained with Liu's stain. The total filter membrane was divided into six fields. Each field was randomly photographed and counted. ${ }^{16}$

\section{Platelet adhesion on endothelial cells}

Isolated platelets from the blood bank were fixed in $4 \%$ glutaraldehyde solution for 1 minute at $2^{\circ} \mathrm{C}-8^{\circ} \mathrm{C}$. After a PBS wash, platelets were stained in Sudan Black B solution (Sigma) by immersion for 5 minutes. After staining, platelets were washed with PBS and at $1,200 \times g$ for 5 minutes. Platelets were counted using a hemacytometer and resuspended at a concentration of $5 \times 10^{7}$ platelets $/ \mathrm{mL}$ in PBS. This platelet suspension $(200 \mathrm{~mL} /$ well) was added in a 24-well plate. Platelets were then allowed to adhere to confluent endothelial cells at $37^{\circ} \mathrm{C}$ for 30 minutes. Later, nonadherent platelets were removed by a gentle wash with PBS. Adherent platelets stained with Sudan Black B were extracted with $200 \mathrm{~mL}$ DMSO/well. After 20 minutes on a shaker, the $\mathrm{OD}_{595}$ was recorded on a microplate reader spectrophotometer.

\section{Nitric oxide (NO) measurement}

Intracellular NO levels were evaluated with 4, 5-diaminofluorescein diacetate (DAF-2DA) staining. Briefly, HUVECs were cultured on six-well plates and grown under $90 \%$ confluence. HUVECs were then pretreated with or without $\mathrm{N}^{\mathrm{G}}$-monomethyl-L-arginine (L-NAME; $1 \mathrm{mmol} / \mathrm{L}$ ) for 30 minutes. PTU ( $5 \mathrm{mmol} / \mathrm{L})$ was then added to HUVECs for 2 hours and incubated with $10 \mu \mathrm{mol} / \mathrm{L}$ DAF-2DA for 30 minutes at $37^{\circ} \mathrm{C}$. Fluorescence was measured using a confocal microscope (Leica TCS SP2, Wetzlar, Germany) with excitation at 488-nm argon laser, and emission was recorded using a long-pass $>530$-nm filter set to acquire twodimensional images $(512 \times 512$ pixel). The relative change of fluorescence intensity was processed with MetaMorph.

\section{Co-immunoprecipitation}

After 24 hours of serum starvation, HUVECs were treated with or without PTU ( $5 \mathrm{mmol} / \mathrm{L}$ ) for 24 hours. The cells were harvested with lysis buffer $(25 \mathrm{mmol} / \mathrm{L}$ Tris- $\mathrm{HCl}$ [pH 7.6], $0.3 \mathrm{~mol} / \mathrm{L} \mathrm{NaCl}, 1.5 \mathrm{mmol} / \mathrm{L} \mathrm{MgCl}_{2}, 0.2 \mathrm{mmol} / \mathrm{L}$ EDTA, $0.5 \%$ Nonidet P-40, and $0.5 \mathrm{mmol} / \mathrm{L}$ dithiothreitol) and immunoprecipitated with either anti-eNOS (BD \#610299) or anti-heat shock protein (hsp)- 90 antibody (Cell Signaling Technology \#4874). After incubation with $25 \mu \mathrm{L}$ protein G-agarose beads for 1 hour, beads were collected, washed three times, and resuspended in SDS sample buffer. The immunocomplexes were resolved by SDS-PAGE and analyzed by Western blotting analysis with either anti-hsp 90 or anti-eNOS antibody.

\section{Statistics and data analysis}

All data are presented as mean \pm standard error (SE). Differences between two groups were tested using unpaired $t$-tests (for continuous variables normally distributed), or Mann-Whitney $U$-tests (for continuous variables that violated the assumption of normal distribution). For multiple groups without repeated measures, one-way ANOVA with the post hoc Scheffe test was used. Two-way ANOVA models, followed by post hoc Bonferroni test, were applied to compare VSMCs and endothelial cells. Differences were considered statistically significant at $p$-value $<0.05$. 
SPSS version 17.0 (for Windows; SPSS Inc, Chicago, IL, USA) was used for data analysis.

\section{Results}

\section{In vitro assessment of hybrid stent/ biodegradable nanofibers}

This study used electrospinning to fabricate hybrid stents and generate PTU-loaded nanofibers (Figure 1A). The diameters of the electrospun PLGA/PTU nanofibers ranged from 112 to $622 \mathrm{~nm}$, with a mean diameter of 246 $\pm 104 \mathrm{~nm}$ (Figure 1B).

The in vitro release behavior of the hybrid stents/drug-eluting nanofibers was determined by an elution method and an HPLC assay. The daily release of PTU from the nanofibers (Figure 2A) and its accumulated release curves (Figure 2B) were plotted. The hybrid stents exhibited a biphasic release pattern with an initial burst release for the first 4 days followed by a rather stable daily release $(6-12 \mu \mathrm{g} / \mathrm{mL})$ until $\sim 3$ weeks.

\section{In vitro observation of platelet adhesion in the stent}

The influence of PTU-loaded nanofibers on platelet adhesion was examined in vitro. Figure 3 displays the photographs of the nanofibers to which platelets had adhered. Platelets adherence to the nanofibers loaded with $25 \mu \mathrm{g} / \mathrm{mm}^{2}$ PTU was significantly lesser than to those without PTU loading $\left(4,800 \pm 1,671 / \mathrm{mm}^{2}\right.$ vs $\left.23,600 \pm 4,558 / \mathrm{mm}^{2}, p<0.001\right)$ after 3 hours of platelet exposure (Figure 3 ).

\section{In vivo animal studies}

All animals survived and had no evidence of infection during the in-life phase of the study. PTU-treated vessels had much thinner intima than controls $(47 \pm 4.9 \mu \mathrm{m}$ vs $288 \pm 22 \mu \mathrm{m}$, $p=0.01$; Figure 4 ). The vascular inflammation and injury scores were comparable in both groups at Week 8 after stent implantation (inflammation in Group A: 0.23 \pm 0.12 , Group B: 0.25 \pm 0.11 ; injury score in Group A: 1.45 \pm 0.25 , Group B: $1.46 \pm 0.22$, respectively). Furthermore, the serum level of TSH was similar for both groups at Week 8 (Group A: 9.4 $\pm 0.5 \mu \mathrm{IU}$; Group B: $8.4 \pm 0.9 \mu \mathrm{IU}, p=0.22$ ), excluding the systemic effect of PTU during local application.

The endothelial coverage was observed and is shown in Figure 5. At Week 3, re-endothelialization onto the surface of struts was variable in both groups, but with significantly greater coverage in Group A $(97.8 \% \pm 1.4 \%)$ than in Group B $(85.2 \% \pm 3.3 \% ; p<0.05)$.

\section{Effect of PTU on endothelial cell function}

Because our in vivo data indicated that PTU might have a protective effect on re-endothelialization, the next experiments were designed to evaluate the effect of PTU on endothelial cell function in vitro. Firstly, the inhibitory effects of PTU on proliferation and migration were more prominent in VSMCs than in endothelial cells (Figure 6). Furthermore, treatment of endothelial cells with PTU reduced the expression of the adhesion molecule (PECAM-1;17 Figure 7A), which translated to decreased platelet adhesion at the surface of confluent endothelial cells (Figure 7B). Finally, it has been indicated that endothelium-derived NO may suppress platelet adhesion and be recognized as a crucial marker of endothelial cell function. ${ }^{18}$ Although our previous study has shown that PTU could promote NO production in isolated rat aortas, ${ }^{19}$ we next determined whether the relevant findings were verified in cultured endothelial cells, especially focusing on eNOS activity. Generally, eNOS synthesizes $\mathrm{NO}$ and its activity is regulated by reversible phosphorylation and the association with other proteins, such as hsp90. ${ }^{20-22}$
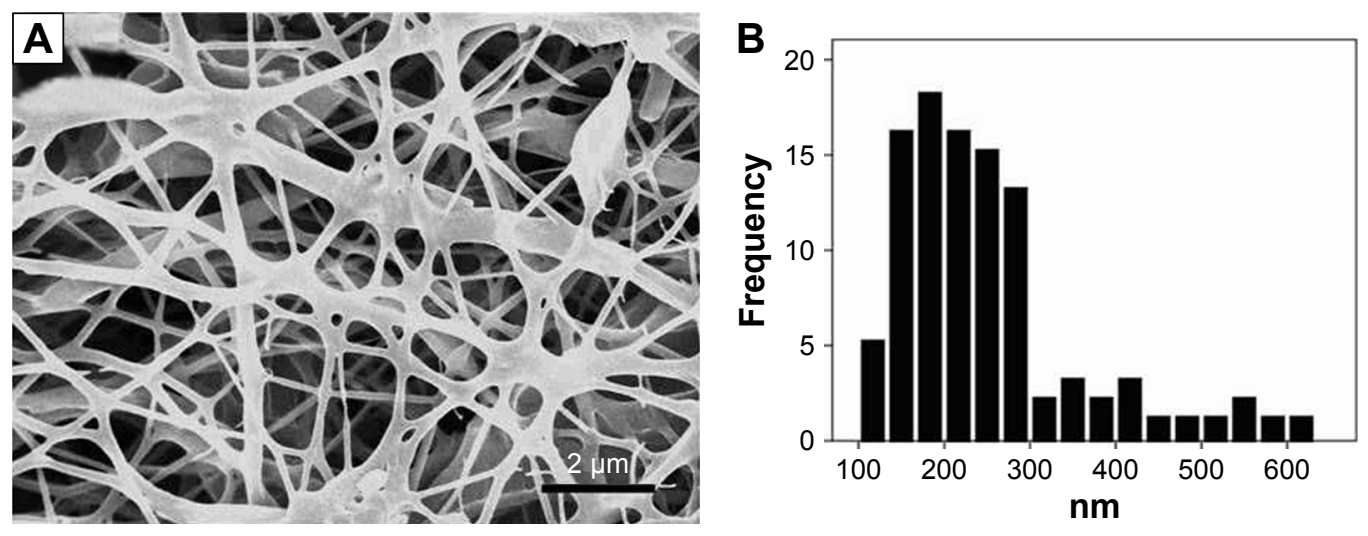

Figure I Morphology of nanofibrous membrane illustrated by scanning electron microscopy.

Notes: Electrospun PLGA/PTU nanofibers is shown (A); magnification $\times 5000$. The diameters of the electrospun PLGA/PTU nanofibers ranged from I I 2 to 622 mm (B). Abbreviations: PLGA, poly(D,L)-lactide-co-glycolide; PTU, propylthiouracil. 
A

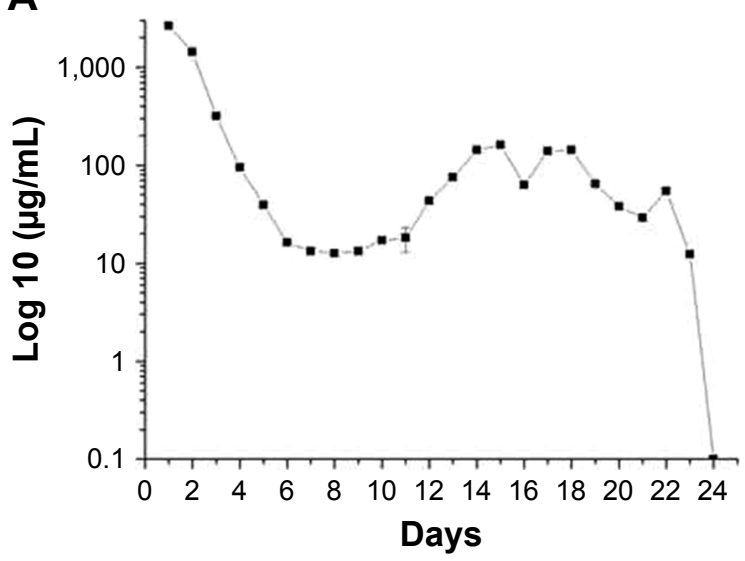

B

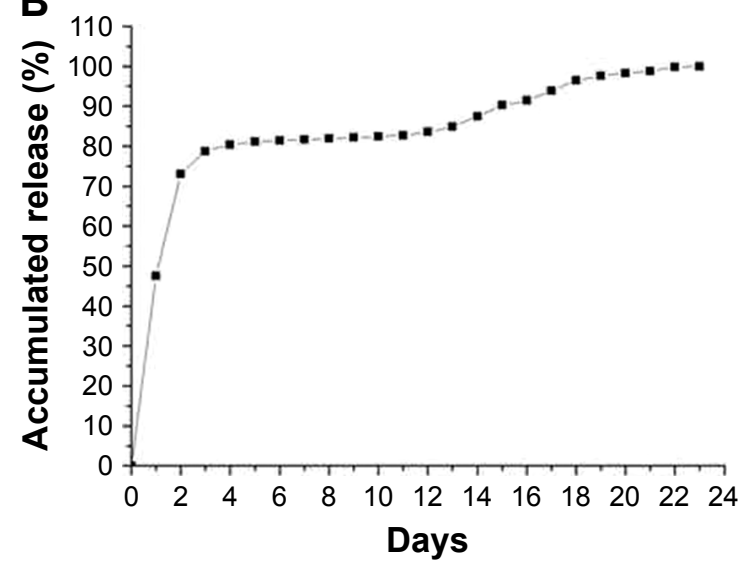

Figure 2 In vitro release of PTU.

Notes: In vitro daily $(\mathbf{A})$ and accumulated release $(\mathbf{B})$ of PTU from the nanofibrous membrane. Each value represents the mean \pm SE of three independent experiments. Abbreviations: PTU, propylthiouracil; SE, standard error.

Consistently, we found that PTU increased the production of NO (Figure 8A), enhanced the phosphorylation of eNOS (Ser1177; Figure 8B and C), and triggered an interaction between eNOS and hsp90 in endothelial cells (Figure 8D).

\section{Discussion}

We have successfully manufactured PTU-eluting stents, implanted them in injured aortas of rabbits, and maintained a stable release of PTU for 3 weeks. This study has some novel findings. Firstly, PTU-coated stent markedly decreased neointimal formation induced by vascular injury. Secondly, the PTU coating reduced platelet adhesion on the surface of biodegradable membrane, which was reflected by the decreased expression of adhesion molecule in PTU-treated endothelial cells. Thirdly, the PTU coating enhanced re-endothelialization of the injured aorta. Fourthly, PTU exerted a less suppressive effect on endothelial cell proliferation and migration, which may provide better
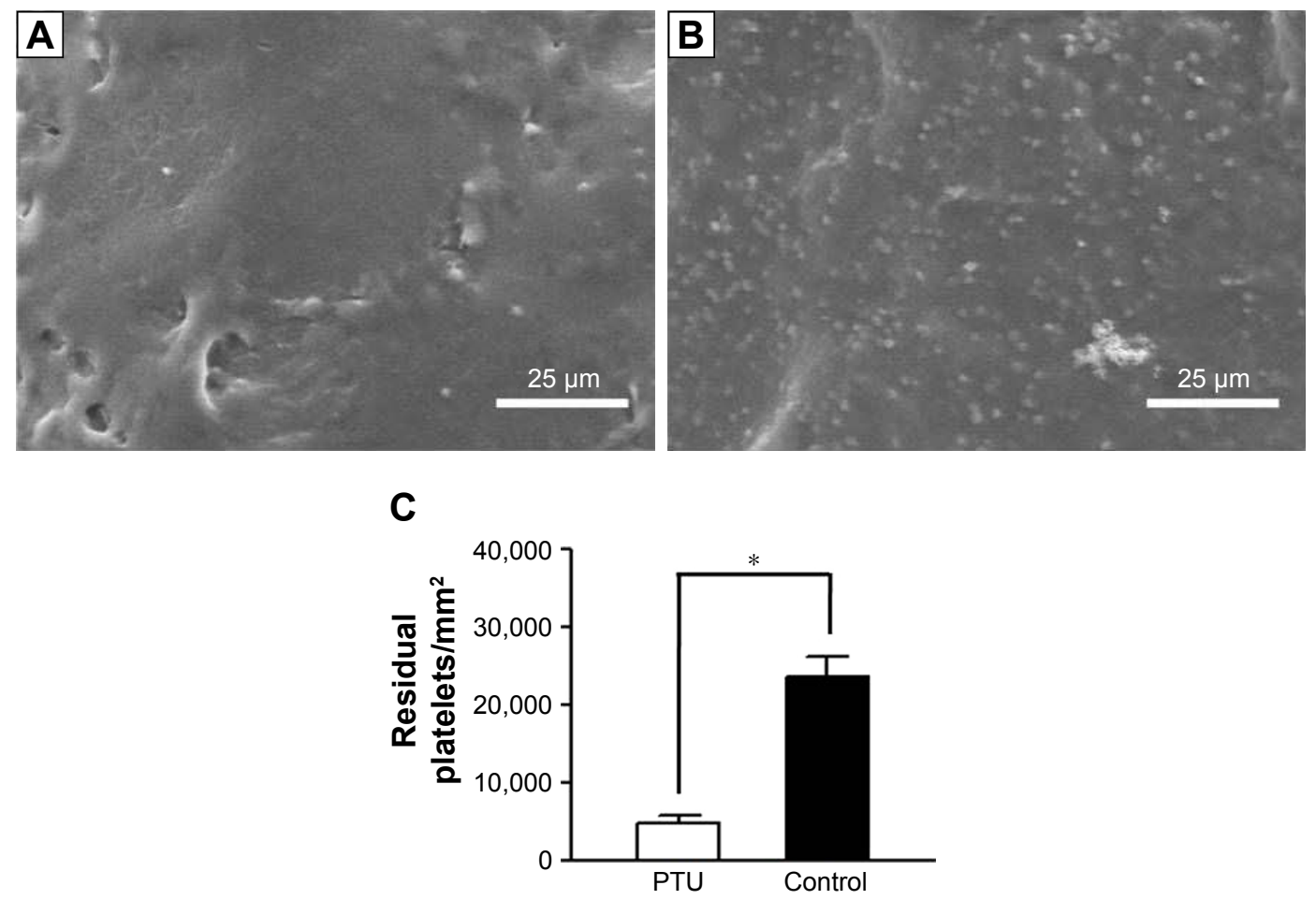

Figure 3 Platelet adhesion test in vitro.

Notes: Fabricated nanofibers with (A) or without (B) a PTU loading $\left(25 \mu \mathrm{g} / \mathrm{mm}^{2}\right)$ were immersed in platelet-rich plasma for 3 hours, and the platelets adherent to the fibers were then counted and averaged $(\mathbf{C})$. Magnification $\times 500$. Each value represents the mean \pm SE of four independent experiments. *Represents significant difference. Abbreviations: PTU, propylthiouracil; SE, standard error. 

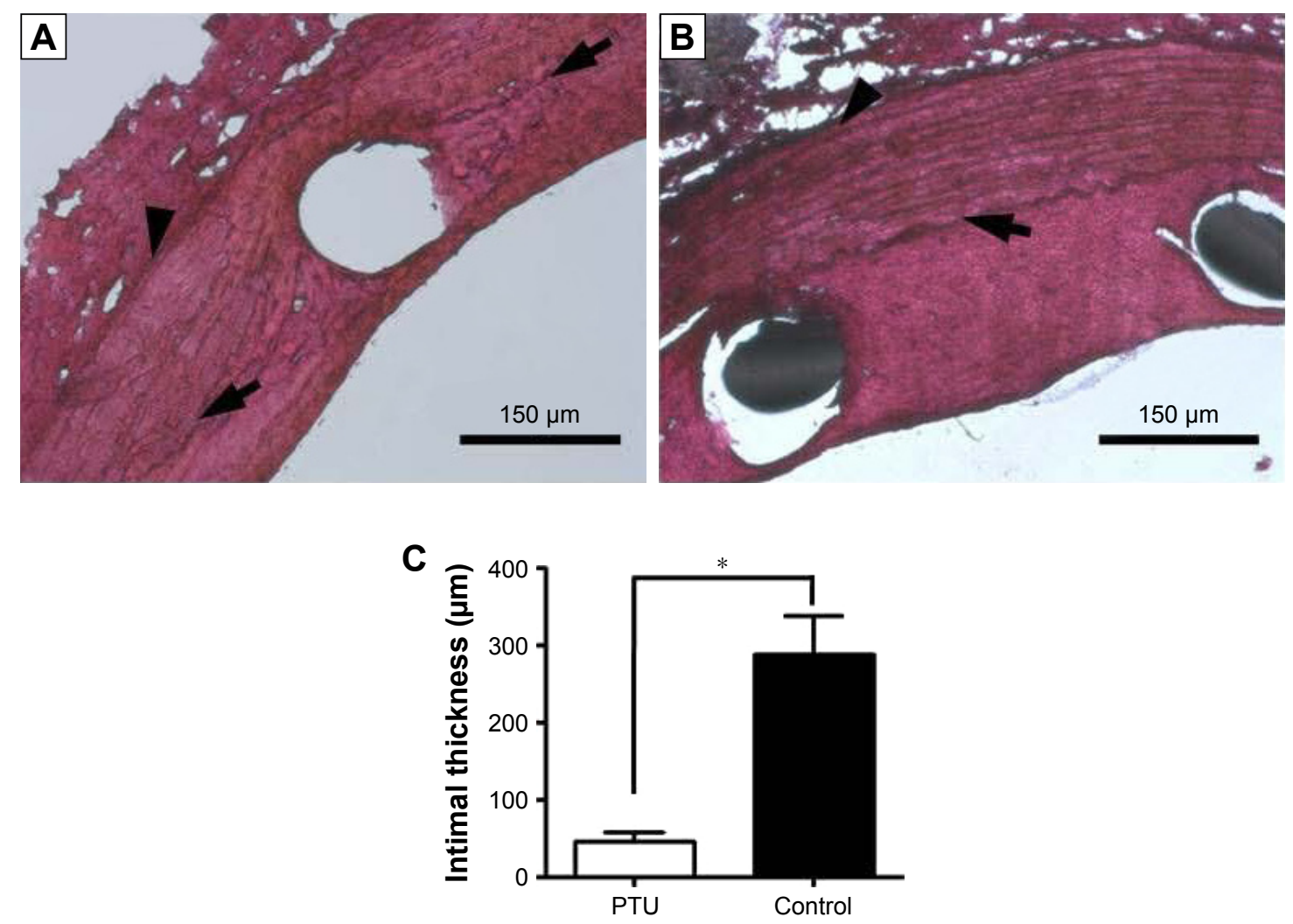

Figure 4 Neointimal formation in the descending aortas of rabbits after intervention.

Notes: Representative photomicrographs of rabbit aortas with (A) or without (B) PTU loading $\left(25 \mu \mathrm{g} / \mathrm{mm}^{2}\right)$ at Week 8 are shown. Magnification $\times 100$. Intimal thickness was counted and averaged (C). Each value represents the mean \pm SE of measurements from six rabbits. *Represents significant difference. Arrows denote the internal elastic lamina, and arrowheads denote the external elastic lamina.

Abbreviations: PTU, propylthiouracil; SE, standard error.
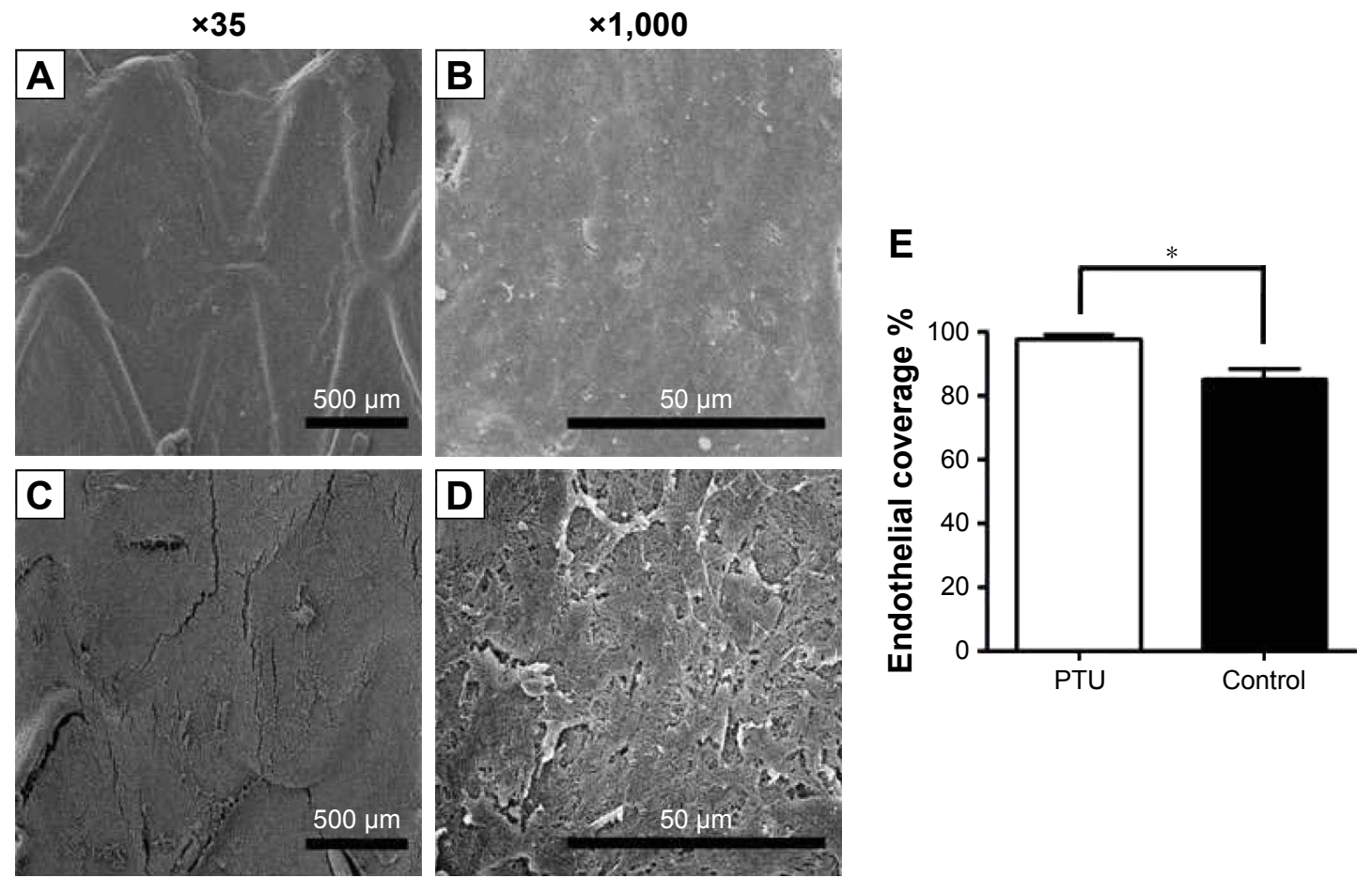

Figure 5 Endothelial coverage and morphology of the endothelial cell layer.

Notes: Endothelial coverage on stent struts with (A and B) or without (C and D) PTU loadings $\left(25 \mu \mathrm{g} / \mathrm{mm}^{2}\right)$ at Week 3 is shown. Magnification $\times 500$. Percentage of endothelial coverage on stent strut was counted and averaged $(E)$. Each value represents the mean \pm SE of measurements from six rabbits. *Represents significant difference. Abbreviations: PTU, propylthiouracil; SE, standard error. 


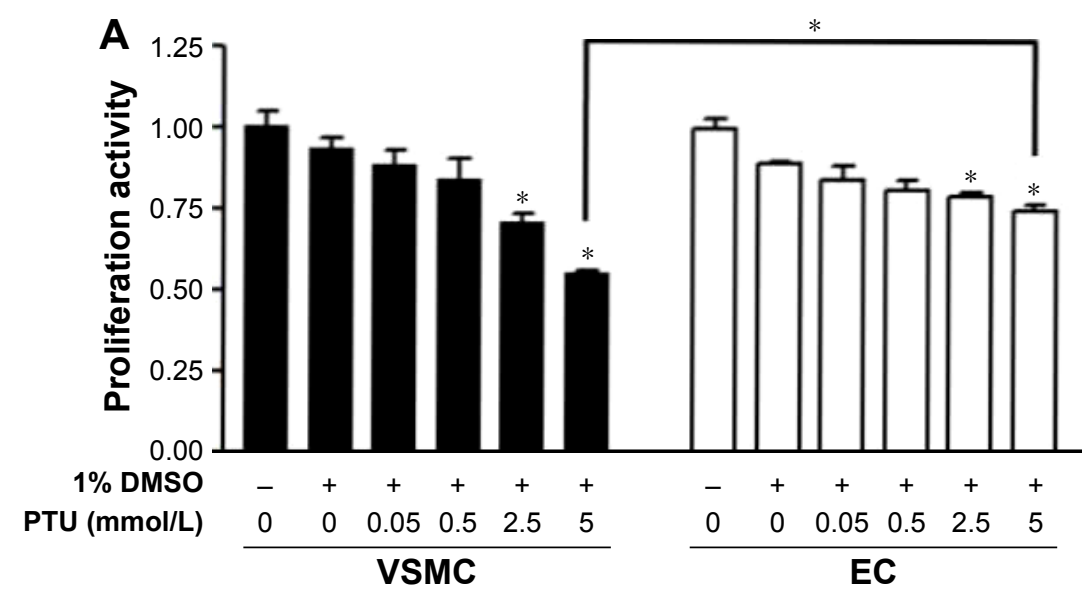

B VSMC
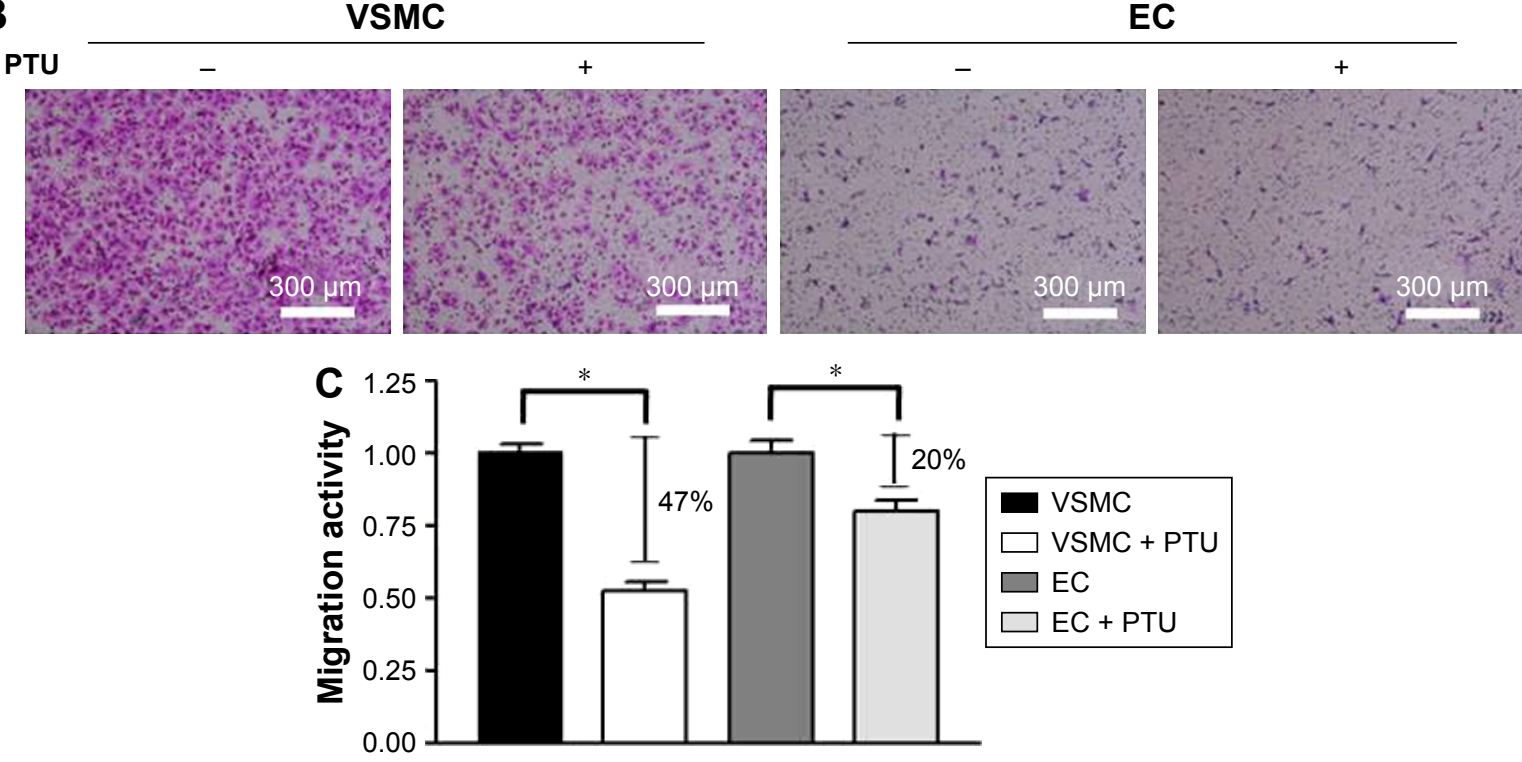

Figure 6 Effect of PTU on proliferative and migratory activities of VSMCs and endothelial cells.

Notes: (A) Proliferative activity was assessed by BrdU incorporation assay. After 24 hours of serum deprivation, cells were treated with indicated conditions for 16 hours. BrdU incorporation into cells was assayed. Each value represents the mean \pm SE of six independent experiments. $p<0.05$; *represents significant difference among groups. (B) Migratory activity was assessed by using a migration assay chamber. After treatment with or without 5 mmol/L PTU for 6 hours, cells on the lower aspect of the filter membrane were fixed and stained with Liu's stain (pink staining; magnification I00x). (C) Each value (mean \pm SE [ $n=6$ ]) is determined by cell number on the lower aspect of filter membrane and expressed as a percentage of control. *Represents significant difference.

Abbreviations: EC, endothelial cells; VSMC, vascular smooth muscle cells; PTU, propylthiouracil; SE, standard error.

endothelial protection. Finally, PTU could not only promote NO production but also induce eNOS activation, supporting the protective role of PTU in endothelial function. Taken together, these findings imply that the local release of PTU by intravascular stent may suppress neointimal formation after vascular injury, accelerate vascular repair after barotrauma, and prevent acute thrombosis at the stenting lesion.

This is the first study to demonstrate the beneficial effect of locally released PTU - a medication used to treat hyperthyroidism - on vascular response to injury or stenting. Our previous studies show that PTU possesses an inhibitory effect on VSMC proliferation and migration, ${ }^{8}$ both of which may contribute to the pathogenesis of atherosclerosis and neointimal formation. This study further confirms that this effect seems to be comparable with both systemic administration and local application. These findings provide a valuable implication that PTU may prevent restenosis after balloon injury by coating of the stent. This approach may successfully prohibit the systemic hypothyroid effect of PTU.

Furthermore, this study showed the inhibitory effect of PTU on platelet adhesion in vitro. Although PTU usage is associated with thrombocytopenia via various possible mechanisms, ${ }^{23}$ the involvement of PTU in platelet adhesion has never been reported. The adhesion of platelets on the endothelium plays an important role in triggering acute thrombosis after angioplasty. ${ }^{3,4,24}$ Although the exact 


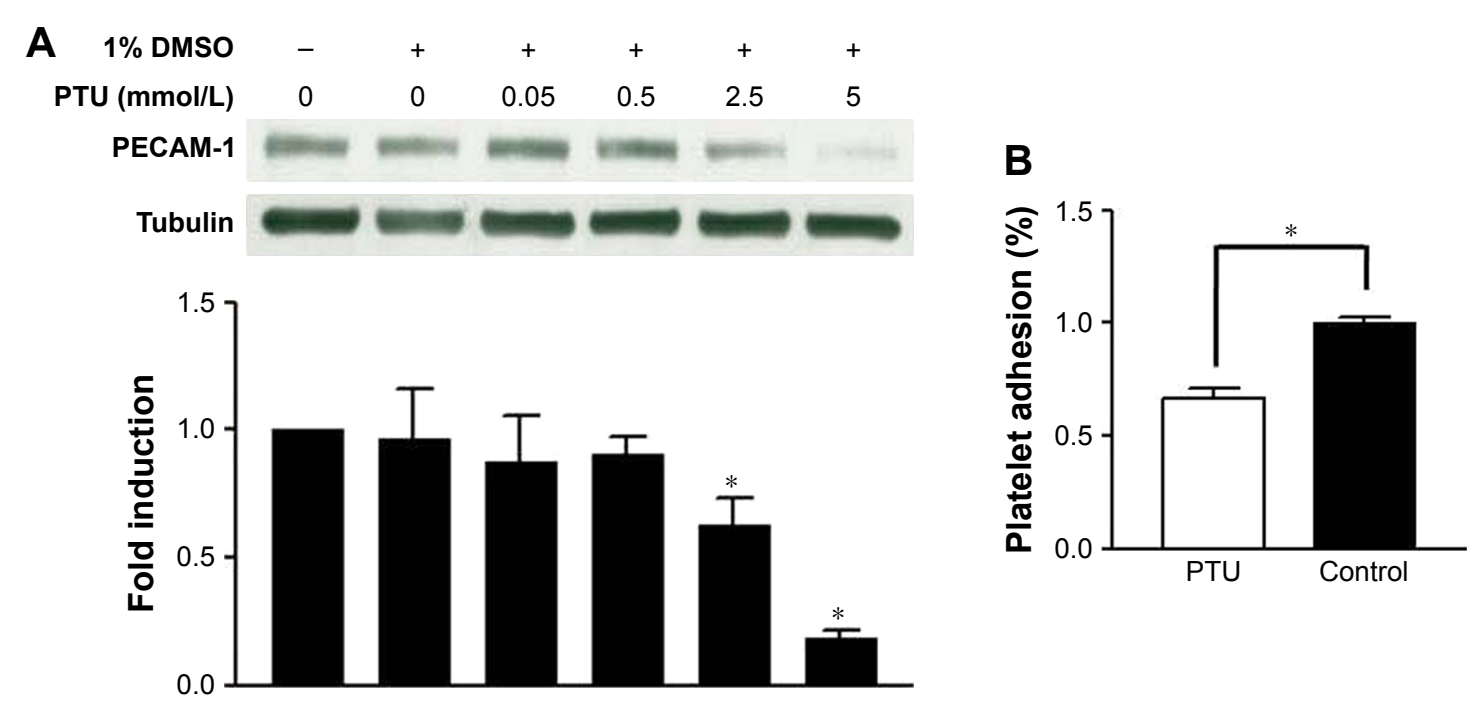

Figure 7 Effect of PTU on adherent activity of endothelial cells.

Notes: (A) After 24 hours of serum deprivation, human umbilical vein endothelial cells were treated with the indicated conditions for 24 hours. The level of PECAM-I was analyzed by Western blotting analysis using tubulin as an internal control. The relative expression level of PECAM-I was quantified by densitometry and normalized to the control level, which was set at I.0. Each value represents the mean \pm SE of four independent experiments. $p<0.05$; *represents significant difference among groups. (B) Confluent endothelial cells were immersed in platelet-rich plasma for $\mathbf{3 0}$ minutes, and the platelets adhered to human umbilical vein endothelial cells were then counted and averaged. Each value represents the mean $\pm S E$ of four independent experiments. *Represents significant difference.

Abbreviations: DMSO, dimethysulfoxide; PECAM-I, platelet and endothelial cell adhesion molecule-I; PTU, propylthiouracil; SE, standard error.

A
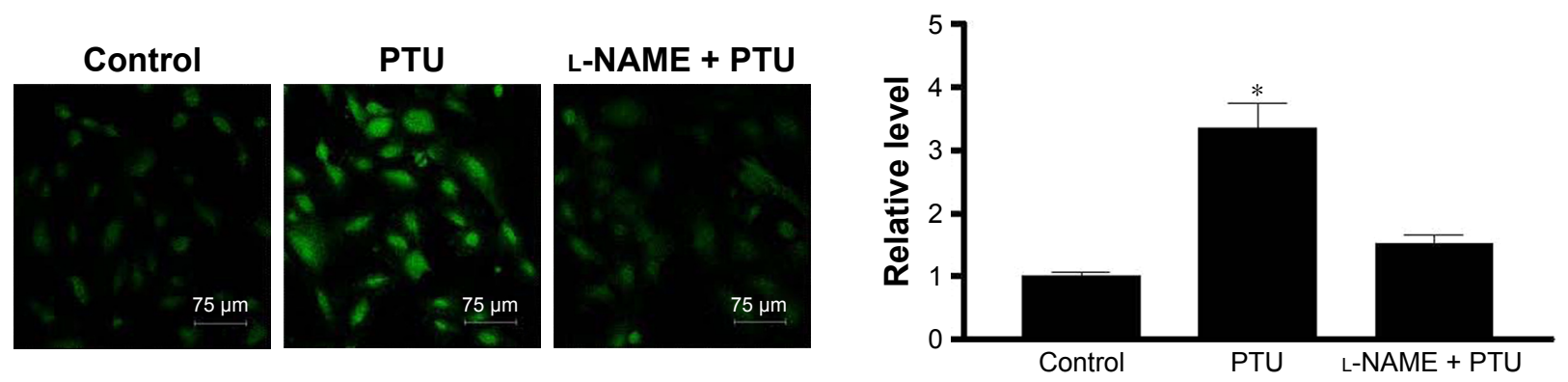

B

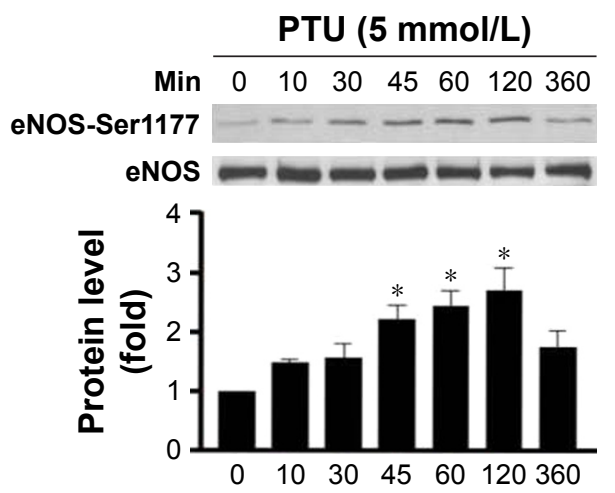

C

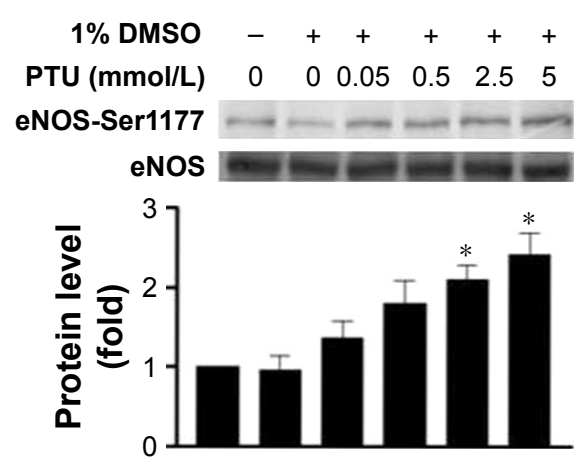

D
(5 mmol/L) control

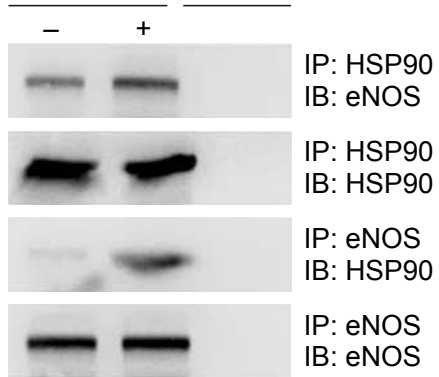

Figure 8 Effect of PTU on NO/eNOS activity of endothelial cells.

Notes: (A) Intracellular NO levels were evaluated with DAF-2DA staining. After 24 hours of serum deprivation, human umbilical vein endothelial cells were seeded on six-well plates and treated with or without $5 \mathrm{mmol} / \mathrm{L}$ PTU in the presence or absence of L-NAME. Fluorescence was measured and the relative change of fluorescence intensity was determined as described in the "Materials and methods" section. (B) After 24 hours of serum deprivation, human umbilical vein endothelial cells were treated with $5 \mathrm{mmol} / \mathrm{L}$ PTU for the indicated durations. The levels of p-eNOS (Serl I77) were analyzed by Western blotting using total eNOS as an internal control. (C) After 24 hours of serum deprivation, human umbilical vein endothelial cells were treated with indicated concentrations of PTU for I20 minutes. The relative expression level of p-eNOS (Serl I77) was quantified by densitometry and normalized to the control level, which was set at I.0. Each value represents the mean \pm SE of four independent experiments. $p<0.05$; *represents significant difference among groups. (D) Co-immunoprecipitation was undertaken using either anti-eNOS or anti-hsp 90 antibody to immunoprecipitate cell lysates from growth-arrested human umbilical vein endothelial cells treated with or without $5 \mathrm{mmol} / \mathrm{L}$ PTU for 24 hours. The immunocomplexes were resolved by Western blotting with either anti-hsp90 or anti-eNOS antibody. The picture is a representative of three blots from three independent experiments.

Abbreviations: DMSO, dimethysulfoxide; IB, immunoblotting; IP, immunoprecipitation; PTU, propylthiouracil; SE, standard error; L-NAME, NG-monomethyl-L-arginine; p-eNOS, phospho-endothelial nitric oxide synthase. 
mechanisms behind how and why PTU affects platelet functions are still unknown, there are some possible explanations for this effect. Firstly, our present and previous studies show that PTU could enhance the production of $\mathrm{NO}^{19}-\mathrm{a}$ molecule reported to inhibit leukocyte/platelet-endothelium adhesion $^{18,25}$ - in the rat aorta. Secondly, although we have found decreased expression of the adhesion molecule in PTU-treated endothelial cells, further studies are needed to evaluate the effect of PTU on other platelet functions such as aggregation. Finally, PTU is an anti-inflammatory agent that is beneficial for its treatment in Graves disease. ${ }^{26}$ Although we could not demonstrate the inhibitory effect of PTU on inflammation at the stenting region, the contribution of this anti-inflammatory action to the PTU-inhibited platelet adhesion may be also considered. Conceivably, our findings implicate that the PTU-coated stent may provide the beneficial effects of shorter antiplatelet usage and lower bleeding risk after vascular intervention.

Another important finding in this study is that the PTUcoated stent could promote re-endothelialization in injured rabbit aortas. The fact that the suppressive effect of PTU on proliferation and migration is more potent on VSMCs than on endothelial cells may partially explain this action of PTU. Furthermore, because the increase of NO/eNOS activity might result in accelerated endothelialization, ${ }^{18,25,27}$ a PTU-coated stent might act as an NO donor to provoke endothelialization. Finally, because inflammation at the balloon-injured site may hinder endothelialization, ${ }^{4}$ the possible involvement of the anti-inflammatory action of PTU in its effect on endothelialization may be taken into account. Due to the fact that early endothelialization may also prevent platelet adhesion and aggregation, ${ }^{4,28}$ our findings provide further evidence that PTU coating of stents may have the advantage of shortening antiplatelet therapy during clinical application.

The local concentration of PTU at the stenting region (6-12 $\mu \mathrm{g} / \mathrm{mL}$ ) falls within the blood concentration of humans who take a therapeutic dose of 300-600 mg daily (1-10 $\mu \mathrm{g} / \mathrm{mL} ; ~ 0.006-0.06 \mathrm{mmol} / \mathrm{L}) .{ }^{29}$ Conceivably, a PTUcoated stent may exert a local effect during clinical application. However, the concentration of PTU (1-10 mmol/L) used in vitro is much higher than the blood concentration. Our explanation for this discrepancy is that it may just reflect the acute effect of PTU on cultured cells.

This study has some limitations. Firstly, the function of platelet and the activity of NO/eNOS were not measured in vivo. The exact role of PTU in platelet function at lesion sites is still unknown. Secondly, denuded rabbit aortas without atherosclerosis were not equal to real lesions in humans. Thirdly, we did not undertake parallel comparisons between sirolimus - a traditional drug used in the clinic to coat stents ${ }^{1,2}$ - and PTU to evaluate their effects on endothelial cell and platelet functions. Finally, the follow-up period of 8 weeks may be too short to estimate the degree of restenosis or thrombosis. Further study is need to evaluate the long-term effect of PTU-coated stents on restenosis and thrombosis.

In conclusion, this study indicates that PTU can be released locally and steadily at injured arteries with some local effects but without systemic effects or side effects. Additionally, PTU may have some beneficial effects on neointimal formation, endothelial cell, and platelet function. We plan to investigate the long-term effect of locally released PTU on the prevention of restenosis and acute thrombus in large animals, which may potentially lead to a clinical trial in humans.

\section{Acknowledgments}

We thank Mr Chih-Chun Chen for his technical assistance in confocal microscopy. This work was supported by grants from the Chang Gung Research Grant Foundation (CMRPG3E102).

\section{Disclosure}

US patent $(6,939,863)$ using PTU for the prevention of atherosclerosis and restenosis has been licensed by Wei-Jan Chen. The authors report no other conflicts of interest in this work.

\section{References}

1. Pursnani S, Korley F, Gopaul R, et al. Percutaneous coronary intervention versus optimal medical therapy in stable coronary artery disease: a systematic review and meta-analysis of randomized clinical trials. Circ Cardiovasc Interv. 2012;5(4):476-490.

2. Yokoyama S, Takano M, Yamamoto M, et al. Extended follow-up by serial angioscopic observation for bare-metal stents in native coronary arteries: from healing response to atherosclerotic transformation of neointima. Circ Cardiovasc Interv. 2009;2(3):205-212.

3. Finn AV, Joner M, Nakazawa G, et al. Pathological correlates of late drug-eluting stent thrombosis: strut coverage as a marker of endothelialization. Circulation. 2007;115(18):2435-2441.

4. Inoue T, Croce K, Morooka T, Sakuma M, Node K, Simon DI. Vascular inflammation and repair: implications for re-endothelialization, restenosis, and stent thrombosis. JACC Cardiovasc Interv. 2011;4(10): $1057-1066$.

5. Kimura T, Morimoto T, Nakagawa Y, et al; j-Cypher Registry Investigators. Antiplatelet therapy and stent thrombosis after sirolimus-eluting stent implantation. Circulation. 2009;119(7):987-995.

6. Giustino G, Baber U, Sartori S, et al. Duration of dual antiplatelet therapy after drug-eluting stent implantation: a systematic review and meta-analysis of randomized controlled trials. $J$ Am Coll Cardiol. 2015;65(13):1298-1310. 
7. Palmerini T, Bacchi Reggiani L, Della Riva D, et al. Bleeding-related deaths in relation to the duration of dual-antiplatelet therapy after coronary stenting. J Am Coll Cardiol. 2017;69(16):2011-2022.

8. Chen WJ, Lin KH, Lai YJ, Yang SH, Pang JH. Protective effect of propylthiouracil independent of its hypothyroid effect on atherogenesis in cholesterol-fed rabbits: PTEN induction and inhibition of vascular smooth muscle cell proliferation and migration. Circulation. 2004;110(10):1313-1319.

9. Chen WJ, Pang JH, Lin KH, Yang SH. Propylthiouracil, independent of its antithyroid effect, decreases VSMC collagen expression. Basic Res Cardiol. 2009;104(1):60-68.

10. Chen WJ, Pang JH, Lin KH, Lee DY, Hsu LA, Kuo CT. Propylthiouracil, independent of its antithyroid effect, promotes vascular smooth muscle cells differentiation via PTEN induction. Basic Res Cardiol. 2010; 105(1):19-28.

11. Lee $\mathrm{CH}$, Chang $\mathrm{SH}$, Lin $\mathrm{YH}$, et al. Acceleration of re-endothelialization and inhibition of neointimal formation using hybrid biodegradable nanofibrous rosuvastatin-loaded stents. Biomaterials. 2014;35(15): 4417-4427.

12. Schneider CA, Rasband WS, Eliceiri KW. NIH Image to ImageJ: 25 years of image analysis. Nat Methods. 2012;9(7):671-675.

13. Ruiter MS, van Tiel CM, Doornbos A, et al. Stents eluting 6-mercaptopurine reduce neointima formation and inflammation while enhancing strut coverage in rabbits. PLoS One. 2015;10(9):e0138459.

14. Giordano A, Romano S, Monaco M, et al. Differential effect of atorvastatin and tacrolimus on proliferation of vascular smooth muscle and endothelial cells. Am J Physiol Heart Circ Physiol. 2012;302(1): H135-H142.

15. Chen WJ, Chen YH, Lai YJ, et al. GT-repeat length polymorphism in heme oxygenase-1 promoter determines the effect of cilostazol on vascular smooth muscle cells. Int J Cardiol. 2016;222:407-415.

16. Lai YJ, Chang GJ, Yeh YH, Pang JH, Huang CC, Chen WJ. Propylthiouracil attenuates experimental pulmonary hypertension via suppression of Pen-2, a key component of gamma-secretase. PLoS One. 2015; 10(9):e0137426.

17. Chistiakov DA, Orekhov AN, Bobryshev YV. Endothelial PECAM-1 and its function in vascular physiology and atherogenic pathology. Exp Mol Pathol. 2016;100(3):409-415.

18. Moncada S. Nitric oxide in the vasculature: physiology and pathophysiology. Ann N Y Acad Sci. 1997;811:60-67; discussion 67-69.
19. Chen WJ, Ho WJ, Chang GJ, et al. Propylthiouracil, independent of its antithyroid effect, produces endothelium-dependent vasodilatation through induction of nitric oxide bioactivity. Atherosclerosis. 2008; 196(1):383-390.

20. Dimmeler S, Fleming I, Fisslthaler B, Hermann C, Busse R, Zeiher AM. Activation of nitric oxide synthase in endothelial cells by Akt-dependent phosphorylation. Nature. 1999;399(6736):601-605.

21. Fulton D, Gratton JP, McCabe TJ, et al. Regulation of endotheliumderived nitric oxide production by the protein kinase Akt. Nature. 1999; 399(6736):597-601.

22. García-Cardeña G, Fan R, Shah V, et al. Dynamic activation of endothelial nitric oxide synthase by Hsp90. Nature. 1998;392(6678):821-824.

23. Peterson ME, Hurvitz AI, Leib MS, Cavanagh PG, Dutton RE. Propylthiouracil-associated hemolytic anemia, thrombocytopenia, and antinuclear antibodies in cats with hyperthyroidism. J Am Vet Med Assoc. 1984;184(7):806-808.

24. Hara H, Nakamura M, Palmaz JC, Schwartz RS. Role of stent design and coatings on restenosis and thrombosis. Adv Drug Deliv Rev. 2006; 58(3):377-386.

25. Vanhoutte PM. Regenerated endothelium and its senescent response to aggregating platelets. Circ J. 2016;80(4):783-790.

26. Bahn RS, Burch HS, Cooper DS, et al. The role of propylthiouracil in the management of Graves' disease in adults: report of a meeting jointly sponsored by the American Thyroid Association and the Food and Drug Administration. Thyroid. 2009;19(7):673-674.

27. Taite LJ, Yang P, Jun HW, West JL. Nitric oxide-releasing polyurethanePEG copolymer containing the YIGSR peptide promotes endothelialization with decreased platelet adhesion. J Biomed Mater Res B Appl Biomater. 2008;84(1):108-116.

28. Hatton MW, Ross B, Southward SM, Timleck-DeReske M, Richardson M. Platelet and fibrinogen turnover at the exposed subendothelium measured over 1 year after a balloon catheter de-endothelializing injury to the rabbit aorta: thrombotic eruption at the late re-endothelialization stage. Atherosclerosis. 2002;165(1):57-67.

29. Mechanick JI, Davies TF. Medical management of hyperthyroidism: theoretical and practical aspects. In: Falk SA, editor. Thyroid Disease. 2nd ed. Philadelphia, PA: Lippincott-Raven; 1997:253-296.
International Journal of Nanomedicine

\section{Publish your work in this journal}

The International Journal of Nanomedicine is an international, peerreviewed journal focusing on the application of nanotechnology in diagnostics, therapeutics, and drug delivery systems throughout the biomedical field. This journal is indexed on PubMed Central, MedLine, CAS, SciSearch $®$, Current Contents $\AA /$ Clinical Medicine,

\section{Dovepress}

Journal Citation Reports/Science Edition, EMBase, Scopus and the Elsevier Bibliographic databases. The manuscript management system is completely online and includes a very quick and fair peer-review system, which is all easy to use. Visit http://www.dovepress.com/ testimonials.php to read real quotes from published authors. 